\section{Optical Pulsations of HZ Herculis}

ON April 15.3 (UT), we attempted to detect optical variations in $\mathrm{HZ}$ Herculis corresponding to its $1.2 \mathrm{~s}$ X-ray pulsation period. No such variations were observed to a limiting magnitude of 23.5 (modulation $0.01 \%$ ).

The instrumentation used was a conventional EMI 6256B phototube with a computerized pulse counting system. Three hour-long sets of data were taken in white light with the Steward Observatory $229-\mathrm{cm}$ telescope. An integration time of $13 \mathrm{~ms}$ was used. The data were analysed using a CooleyTukey fast Fourier transform routine. A frequency resolution of $3 \times 10^{-4} \mathrm{~Hz}$ was obtained over the range 0.7 to $0.9 \mathrm{~Hz}$.

Observations were planned for April 13 to 15 so as to cover the beginning of the "off phase" of the $35 \mathrm{~d}$ X-ray modulation cycle. In this manner we hoped to demonstrate that the optical pulsations (observed by Groth et al. ${ }^{1}$ during the "on phase" April 6 to 7) terminate simultaneously with the X-ray pulsations. Observations were obtained only on April 15, which was probably during the "off phase".

We propose that the observed optical pulses come to us directly from the X-ray source and are merely the low frequency tail of the X-ray spectrum. Our observations, combined with those of Groth et al., are consistent with this hypothesis. Furthermore, if the pulses were echoes of the X-ray source emitted by the atmosphere of the primary, we would not expect the pulses to have the strength $(0.1$ to $0.2 \%)$ observed by Groth et al. ${ }^{1}$. The pulses would be reduced by a factor of 10 by both light travel time across the primary and the atmospheric leak time $(\sim 100 \mathrm{~s}$, ref. 2$)$.

J. S. Scott and P. A. Strittmatter (to be published) have suggested that the $X$ rays are produced by interaction between free-falling protons and electrons expelled from the surface of the X-ray pulsar. A similar suggestion was proposed by Cocke $^{3}$ to explain the high energy $\gamma$ rays from the Crab pulsar. Such a mechanism predicts a spectrum that is flat from soft $X$ rays to the visual, that is, that the flux density in $\mathrm{erg} \mathrm{cm}^{-2}$ $\mathrm{s}^{-1} \mathrm{~Hz}^{-1}$ is constant.

Using emission region magnetic fields of $10^{12}$ gauss, electron densities of $10^{21} \mathrm{~cm}^{-3}$, electron-proton interaction velocities of $V=10^{10} \mathrm{~cm} \mathrm{~s}^{-1}$ and a path length of $10^{6} \mathrm{~cm}$, as suggested by Scott and Strittmatter, together with a free-free absorption coefficient corrected for intense magnetic fields ${ }^{4}$

$$
\kappa_{\mathrm{ff}}=6.7 \times 10^{-18}\left(\lambda^{3} n_{\mathrm{e}}{ }^{2} / V\right)\left(2 \pi m_{\mathrm{e}} / e B \lambda\right)^{2}
$$

we predict a low frequency turnover at about $1 \mu \mathrm{m}$. Observational studies based on this prediction are in progress.

Taking an X-ray flux ${ }^{5}$ of 0.08 photons $\mathrm{cm}^{-2} \mathrm{~s}^{-1} \mathrm{keV}^{-1}$ at $10 \mathrm{keV}$, we compared the flat extrapolation of the spectrum converted to erg $\mathrm{cm}^{-2} \mathrm{~s}^{-1} \mathrm{~Hz}^{-1}$ with typical quarter phase $U B V$ observations of $\mathrm{HZ}$ Her taken at Steward Observatory, and crudely corrected for filter transmission. This yields a predicted percentage pulse of slightly less than $0.4 \%$, which compares favourably with the values obtained by Groth et $a l .{ }^{1}(0.1-0.2 \%)$. We note that various power laws suggested by Ulmer et al. $^{5}$ predict pulsation amplitudes that are far too large, unless a low frequency turnover is assumed.

The success of the flat spectrum hypothesis in predicting the observed pulse height and the absence of strong emission lines in the visual (ref. 2 and G. Gilbert, private communication) render the often discussed hidden soft $\mathrm{X}$-ray heating source (ref. 6, for example) unnecessary.

We thank S. Carswell, P. A. Strittmatter, R. I. Thompson, F. J. Vrba, and J. A. J. Whelan for advice and assistance.

W. J. COCKE

P. HINTZEN

J. S. SCOTT

S. P. WORDEN
Received July 11, 1973.

${ }^{1}$ Groth, E. J., Yeung, S. Z., Papaliolios, C., Pennypacker, C. R., Spada, G., and Middleditch, J., IAU Circ., No. 2523 (1973).

2 Strittmatter, P. A., Scott, J. S., Whelan, J. A., Wickramasinghe, D. T., and Woolf, N. J., Astr. Astrophys., 25, 275 (1973).

${ }^{3}$ Cocke, W. J., Astrophys. J. (in the press).

4 Tsuruta, S., Canuto, V., Lodenquai, J., and Ruderman, M., Astrophys. J., 176, 739 (1972).

5 Ulmer, M. P., Baity, W. A., Wheaton, W. A., and Peterson, L. E., Astrophysics J. Lett., 181, L33 (1973).

6 Pringle, J. E., Nature phys. Sci., 243, 90 (1973).

\section{Positronium Origin of $476 \mathrm{keV}$ Galactic Feature}

LEVENTHAL $^{1}$ has noticed that the $\gamma$-ray spectrum due to the annihilation of positronium, which consists of two $511 \mathrm{keV}$ photons from the singlet state and three photons from the triplet state, produces a spectral feature (line) with an apparent peak at an energy less than $511 \mathrm{keV}$ when viewed with a $\gamma$-ray telescope having a Gaussian energy resolution. With the energy resolution of the telescope ${ }^{2}$ used to detect the $1.8 \times 10^{-3}$ $\mathrm{cm}^{-2} \mathrm{~s}^{-1}$ galactic feature at $476 \pm 24 \mathrm{keV}$, Leventhal ${ }^{1}$ calculates that the observed peak will lie at $490 \mathrm{keV}$. I calculate, moreover, that if the positronium spectrum sits atop a steeply falling continuum due to other sources, as is observed, then the apparent peak can easily fall near $476 \mathrm{keV}$ where it was observed. Furthermore, the actual data $^{2}$ on the observed feature look like a positronium feature, because there are very few (statistically zero) photons (as opposed to pulses) with energies greater than about $511 \mathrm{keV}$, indicating a sudden drop in the spectrum there. I therefore take Leventhal's suggestion seriously and show here that explosive nucleosynthesis is a plausible source of the positrons.

The primary sources of positrons from explosive nucleosynthesis should be ${ }^{56} \mathrm{Co}$, the progenitor of ${ }^{56} \mathrm{Fe}$ with a half life of $77 \mathrm{~d}$, and ${ }^{44} \mathrm{Sc}$, the daughter of $48-\mathrm{yr}^{44} \mathrm{Ti}$ and progenitor of ${ }^{44} \mathrm{Ca}$. Either or both could be the source of sufficient positrons to account for the observed feature. To see this requires an estimate of the rate of synthesis of the parent nuclei. I shall assume as a model that $1.7 \times 10^{9}$ supernova events during galactic history ${ }^{3}$ synthesized the galactic concentrations of ${ }^{56} \mathrm{Fe}$ and ${ }^{44} \mathrm{Ca}$. Then the average supernova yield is $3.0 \times 10^{54}$ atoms of ${ }^{56} \mathrm{Co}$ and $5.6 \times 10^{51}$ atoms of ${ }^{44} \mathrm{Ti}$. The decay of these nuclei after explosive ejection provides the positrons. Leventhal ${ }^{1}$ argued that if the density is less than $10^{-9} \mathrm{~g} \mathrm{~cm}^{-3}$, the emitted positrons will decay primarily through the formation of positronium. If the details of the dynamics are ignored and one merely assumes $m$ solar masses expanding uniformly at an expansion velocity $10,000 \mathrm{~km} \mathrm{~s}^{-1}$, the mean density would be

$$
\bar{\rho} \approx\left(m / 2.1 t^{3}\right) 10^{6} \mathrm{~g} \mathrm{~cm}^{-3}
$$

where $t$ is the expansion time (s). This density is sufficiently rare for the positronium mode to dominate for $t>10^{5} \mathrm{~s}$. The optical depth

$$
\bar{\rho} R \approx\left(\mathrm{m} / 2.1 t^{2}\right) 10^{15} \mathrm{~g} \mathrm{~cm}^{-2}
$$

is less than $10 \mathrm{~g} \mathrm{~cm}^{-2}$ for $t>10^{7} \mathrm{~s}$, very nearly the mean lifetime of ${ }^{56} \mathrm{Co}$. Therefore one can roughly estimate that $1 / \mathrm{e}$ of the positrons produced by ${ }^{56} \mathrm{Co}$ decay form a positronium annihilation that escapes the expanding remnant. Because the positron emitting branch of ${ }^{56} \mathrm{Co}$ is $20 \%$, we anticipate $(0.20 / \mathrm{e})$ $3.0 \times 10^{54}$ visible annihilations per supernova.

The rate of annihilation of these $2.2 \times 10^{53}$ positrons is a question of the astrophysical environment. I shall consider one of the two simplest cases. If the lifetime of the positron against formation of positronium is greater than the average 